\title{
Capabilities of Cluster Analysis in interpretation of 24-Hour Blood Pressure Monitoring Data in Patients with Arterial Hypertension and Left Ventricular Remodeling
}

DOI: 10.17691/stm2015.7.4.15

Received May 11, 2015

S.V. Samoyavcheva, Cardiologist';

V.V. Shkarin, MD, DSc, Head of Therapy Department, Postgraduate Faculty ${ }^{2}$

${ }^{1}$ Railway Clinical Hospital on Gorky Station, Joint Stock Company "Russian Railway", 18 Lenin Avenue, Nizhny Novgorod, 603140, Russian Federation;

${ }^{2}$ Nizhny Novgorod State Medical Academy, 10/1 Minin and Pozharsky Square, Nizhny Novgorod, 603005, Russian Federation

The aim of the investigation was to assess the potential of cluster analysis as an additional method of data analysis for 24-hour blood pressure monitoring (BPM) in patients with both normal geometry and with various types and extents of remodeling of the left ventricle (LV).

Materials and Methods. The investigation included 71 patients, ranging in age from 23 to 71 . The inclusion criterion was significant arterial hypertension $(\mathrm{AH})$, while exclusion criteria were symptomatic $\mathrm{AH}$ and severe co-morbidity. Body mass, height, waist measurement, body mass index, lipid profile, and glycemic level were determined for each subject in addition to carrying out echocardiography and conventional and cluster analysis of 24-hour BPM data of each.

Results. In patient groups with different types of left ventricular hypertrophy (LVH), the conventional analysis demonstrated differences in the standard 24-hour BPM parameters. Development of concentric LVH is associated with the highest average day-time and average night-time blood pressure, pressure-induced loads and blood pressure variability. Eccentric LVH has a pathogenetic link to other factors and is formed under conditions of relatively low blood pressure. The use of cluster analysis allowed to reveal the increased occurrence of systolic-diastolic $\mathrm{AH}$ in concentric LVH, and isolated systolic AH and isolated diastolic AH in eccentric LVH.

Conclusion. Such an integrated approach to the interpretation of 24-hour BPM results, comprising both conventional and cluster analysis, allows for objectification of the study results and reveals the significant features of $\mathrm{AH}$ in patients with different types of LV remodeling.

Key words: 24-hour blood pressure monitoring; 24-hour BPM; left ventricular remodeling; left ventricular hypertrophy.

Arterial hypertension $(\mathrm{AH})$ is an independent risk factor for cardiovascular complications and mortality. The severity of the clinical implications and the prognosis for $\mathrm{AH}$ patients are determined not only by the degree of blood pressure (BP) increase but to a great extent by the presence of any target lesions, including left ventricular hypertrophy $(\mathrm{LVH})[1,2]$.

The risk of complications depends both on the degree of hypertrophy and the type of left ventricular (LV) remodeling. In the case of concentric remodeling, the probability of cardiovascular complications within the next 10 years is $15 \%$; in the case of eccentric LVH it is $25 \%$ and in the case of concentric LVH it is $30 \%$ [2].

There is now evidence of a significant association between LV changes and of the indices revealed by 24-hour blood pressure monitoring (BPM) [3]:

a closer correlation between LV myocardial mass index (LVMMI), LV diastolic function and left atrial dimension with pressure-induced load, than with the clinical and daily mean BP measurements;

a relationship between hypertrophy and abnormal geometry of the LV in $\mathrm{AH}$ with variation in $\mathrm{BP}$; a relationship between increased LVMMI in $\mathrm{AH}$ and a decreased extent of reduction in night-time BP. This indicates that it is the lack of night-time BP reduction that causes organ damage and not vice versa.

However, the above indicators do not reflect all aspects of BP involvement in LV remodeling. Analyses in conventional everyday clinical practice make use of only a few blocks of the large amount of digital information that is available from 24-hour BPM: mean BP values, pressure-induced load indices, BP daily rhythm and variability and morning hemodynamics. As a result, the physician only has a single set of parameters, with each parameter reflecting a specific characteristic of either systolic (SBP) or diastolic blood pressure (DBP). With this approach, the diagnostic opportunities of 24-hour BPM in any particular patient are not used to the full, as no holistic picture of 24-hour regulation and maintenance of BP is produced, and the information about any particular system may be simplified, and sometimes even distorted. In reality, the results of an examination can provide a much greater amount of useful information.

For contacts: Samoyavcheva Svetlana Vladimirovna, e-mail: svsamoyavcheva@gmail.com 
In and of itself the system of BP regulation and maintenance is multidimensional and complex in nature, having a large number of components with a multitude of interactions and dynamic variables. Modern information technology, including the mathematical processing of large amounts of data, offers the opportunity to explore such systems involving a variety of parameters through their representation and systematization within a multidimensional range of attributes.

In order to increase the use of valuable information and to obtain new interpretations and knowledge, it is instructive to apply multi-variance analysis to 24-hour BPM data. Agglomerative hierarchical cluster analysis is one of the principal methods of multi-variance analysis used to resolve classification issues.

The aim of the investigation was to assess the value of cluster analysis as an additional method for analyzing 24-hour blood pressure monitoring data from patients with normal ventricular geometry and those with various types of remodeling.

Materials and Methods. The investigation included 71 patients, aged from 23 to 71 , of which 37 were men $(52.11 \%)$ and 34 were women $(47.89 \%)$. The average age of the patients was $48.89 \pm 12.73$ years. The inclusion criterion was the presence of significant $\mathrm{AH}$, while the exclusion criteria were symptomatic $\mathrm{AH}$ and severe comorbidity.

The study was carried out in accordance with the Declaration of Helsinki (adopted in June 1964 (Helsinki, Finland) and revised in October 2000 (Edinburgh, Scotland)) and approved by the Ethics Committee of the Nizhny Novgorod State Medical Academy. All patients gave their written, informed consent.

Body mass, height, waist measurement and obesity type were determined in all patients; the body mass index (Quetelet index) was calculated using the formula $\mathrm{BMl}=$ body mass $(\mathrm{kg}) /$ height $\left(\mathrm{m}^{2}\right)$; while the lipid profile and glycemic level were analyzed to assess the obesity type.

Following standard procedures, all patients were subjected to 24-hour BPM using a TM-2421 (A\&D Company, Japan) blood pressure monitor, and echocardiography (echoCG) was carried out using a Toshiba 14OA2 (Toshiba, Japan) ultrasonic imaging system.

24-hour BPM was carried out using a mixed auscultatory-oscillometric method against a 3-day nomedication background. The AH level was determined according to the data processing and interpretation software method of Dabl [4]. Based on mean BP values, in 57 patients $(80.28 \%)$ we found degree I $\mathrm{AH}$, in $12(16.9 \%)$ - degree $\mathrm{II} \mathrm{AH}$, and in $2(2.82 \%)-$ degree III $\mathrm{AH}$.

For each patient, their standard 24-hour BPM data were analyzed: average day-time and average nighttime measurements of SBP and DBP, together with the pressure-induced load measurements by time index (TI) of the SBP and DBP, and any variability in the SBP and DBP.

24-hour BPM data cluster analysis was undertaken using Statgraphics Plus 5.0 statistical analysis software and the Ward algorithm. The following clustering parameters were used for each $\mathrm{BP}$ measurement: $\mathrm{SBP}, \mathrm{DPB}$, pulse pressure $\left(\mathrm{BP}_{\mathrm{p}}=\mathrm{SBP}-\mathrm{DBP}\right)$, average pressure $\left(\mathrm{BP}_{\mathrm{av}}=\mathrm{DBP}+1 / 3 \mathrm{BP}_{\mathrm{p}}\right)$, $\mathrm{BP}$ structural point $(\mathrm{BPSP}=\mathrm{DBP} / \mathrm{SBP})$, heart rate $(\mathrm{HR})$. Based on dendrograms, scatter diagrams and their digital characteristics, 24-hour BPM data were stratified into clusters, their quantity was determined, and the percentage ratio of each was calculated. The clusters were then combined into 8 groups: normal and borderline $B P$, isolated systolic $A H$ (ISAH), isolated diastolic AH (IDAH), systolic-diastolic AH (SDAH) all with HRs below 80 per minute, plus corresponding clusters with HRs above 80 per minute.

SBP, DBP and HR were measured prior to echoCG. The echoCG was carried out before noon after the patients had been in a horizontal position for 5 minutes. Measurements were made in the $\mathrm{M}$ - and $\mathrm{B}$-modes using 2.5 and $3.75 \mathrm{MHz}$ sensors. We determined the maximum (LAd) and minimum (LAs) left atrial dimensions, the end-systolic dimension of the LV, the LV dimensions at the end of both rapid and slow filling, the end-diastolic dimension (EDD), the durations of LV systole and diastole, the duration of the rapid and slow filling stages, and the thickness of the interventricular septum (IVS) and the LV posterior wall (LVPW) during systole and diastole.

The calculation of echocardiographic indices was performed using COR software [5]. Using this, we calculated the LV myocardium mass and the LVMMI, according to the Le Verrier and Teichholz formulas [6], plus the end-diastolic and end-systolic volumes, and the stroke volume.

In order to explore the restrictive component of intracardiac hemodynamics, we calculated the atrioventricular ratio $(A V R=L A d / E D D)$. Here, $A V R \geqslant 0.63$ indicates LV restriction.

The Le Verrier formula [6] was used for calculation of the relative wall thickness (RWT) of the LV (RWT=[LVPWd+IVSd]/EDD).

Results and Discussion. Subject to the LVMMI and RWT values, it is common practice to distinguish 4 types of LV geometry [6]:

1) normal geometry (RWT $<0.45$; LVMMI normal);

2) concentric remodeling (RWT $\geqslant 0.45 ; \quad L V M M I$ normal);

3) concentric hypertrophy (RWT $\geqslant 0.45$; LVMMI abnormal);

4) eccentric hypertrophy (RWT $<0.45$; LVMMI abnormal).

According to the LV geometric remodeling type, all the subjects were also divided into 4 further groups. 24-hour BPM and echoCG data are presented in Tables 1-3. 
Table 1

Average values of standard indicators in 24-hour blood pressure monitoring of patients with different left ventricle geometry types

\begin{tabular}{|lccccccc}
\hline Left ventricle geometry type & $\begin{array}{c}\text { Time } \\
\text { of day }\end{array}$ & $\begin{array}{c}\text { SBP } \\
(\mathbf{m m ~ H g})\end{array}$ & $\begin{array}{c}\text { DBP } \\
(\mathbf{m m ~ H g})\end{array}$ & $\begin{array}{c}\text { SBP TI } \\
(\%)\end{array}$ & $\begin{array}{c}\text { DBP TI } \\
(\%)\end{array}$ & $\begin{array}{c}\text { SBPV } \\
(\mathbf{m m ~ H g})\end{array}$ & $\begin{array}{c}\text { DBPV } \\
(\mathbf{m m ~ H g})\end{array}$ \\
\hline \multirow{2}{*}{ Normal $(n=26)$} & Day & 141.95 & 88.25 & 52.10 & 47.01 & 17.49 & 12.03 \\
\hline \multirow{2}{*}{ Concentric remodeling $(\mathrm{n}=10)$} & Night & 120.90 & 72.63 & 49.14 & 31.95 & 10.81 & 7.38 \\
\cline { 2 - 8 } & Day & 147.41 & 89.99 & 62.17 & 56.04 & 20.50 & 12.97 \\
\hline Concentric hypertrophy $(n=18)$ & Night & 123.15 & 71.43 & 63.31 & 21.21 & 9.70 & 7.77 \\
\cline { 2 - 8 } & Day & 148 & 91.33 & 67.71 & 54.61 & 17.58 & 12.47 \\
\hline \multirow{2}{*}{ Eccentric hypertrophy $(n=17)$} & Dight & 125.36 & 76.81 & 66.81 & 40.66 & 9.96 & 8.06 \\
\cline { 2 - 8 } & Night & 123.10 & 74.89 & 59.16 & 28.04 & 9.21 & 7.46 \\
\hline
\end{tabular}

N o t e. SBP and DBP: systolic and diastolic blood pressure; SBP TI and DBP TI: time index of SBP and DBP; SBPV and DBPV: SBP and DBP variability.

Table 2

Occurrence of blood pressure clusters in patients with arterial hypertension and different types of left ventricle geometry

\begin{tabular}{|c|c|c|c|c|c|c|c|c|c|}
\hline \multirow{3}{*}{$\begin{array}{l}\text { Left ventricle } \\
\text { geometry type }\end{array}$} & \multirow{3}{*}{$\begin{array}{l}\text { Time } \\
\text { of day }\end{array}$} & \multicolumn{8}{|c|}{ Occurrence of BP clusters (\%) } \\
\hline & & \multicolumn{2}{|c|}{ Normal BP } & \multicolumn{2}{|c|}{ ISAH } & \multicolumn{2}{|c|}{ IDAH } & \multicolumn{2}{|c|}{ SDAH } \\
\hline & & $H R<80$ & $H R>80$ & $H R<80$ & $H R>80$ & $H R<80$ & $H R>80$ & $H R<80$ & $H R>80$ \\
\hline \multirow[t]{3}{*}{ Normal $(n=26)$} & Day & 16.35 & 10.93 & 4.82 & 9.1 & 3.47 & 5.78 & 11.21 & 16.16 \\
\hline & Night & 10.13 & 0.76 & 1.12 & 0.32 & 1.24 & 0 & 5.56 & 1.21 \\
\hline & 24 hours & 26.48 & 11.69 & 5.94 & 9.42 & 4.71 & 5.78 & 16.77 & 17.37 \\
\hline \multirow{3}{*}{$\begin{array}{l}\text { Concentric remodeling } \\
(n=10)\end{array}$} & Day & 13.94 & 3.28 & 7.18 & 7.74 & 5.14 & 2.71 & 18.76 & 19.24 \\
\hline & Night & 10.84 & 0 & 2.84 & 0 & 1.23 & 0 & 4.91 & 0 \\
\hline & 24 hours & 24.78 & 3.28 & 10.02 & 7.74 & 6.37 & 2.71 & 23.67 & 19.24 \\
\hline \multirow{3}{*}{$\begin{array}{l}\text { Concentric hypertrophy } \\
(n=18)\end{array}$} & Day & 6.8 & 8.42 & 8.19 & 6.31 & 6.2 & 3.22 & 17.8 & 21.25 \\
\hline & Night & 6.92 & 0 & 0.48 & 0.68 & 0.87 & 0.41 & 7.45 & 2.22 \\
\hline & 24 hours & 13.72 & 8.42 & 8.67 & 6.99 & 7.07 & 3.63 & 25.25 & 23.47 \\
\hline \multirow{3}{*}{$\begin{array}{l}\text { Eccentric hypertrophy } \\
(n=17)\end{array}$} & Day & 16.27 & 1.81 & 14.85 & 2.39 & 8.51 & 7.46 & 14.35 & 12.35 \\
\hline & Night & 10.24 & 0 & 0.72 & 0 & 2.33 & 0 & 6.32 & 0 \\
\hline & 24 hours & 26.51 & 1.81 & 15.57 & 2.39 & 10.84 & 7.46 & 20.67 & 12.35 \\
\hline
\end{tabular}

$\mathrm{N}$ o t e. BP: blood pressure; ISAH: isolated systolic arterial hypertension; IDAH: isolated diastolic arterial hypertension; SDAH: systolic-diastolic arterial hypertension; HR: heart rate.

Table 3

Echocardiographic measurements in patients with different left ventricle geometry types

\begin{tabular}{|lccccc}
\hline \multirow{2}{*}{ Left ventricle geometry type } & \multicolumn{2}{c}{ LVMMI } & RWT & AVR & EDD \\
\cline { 2 - 6 } & Men & Women & & & \\
\hline Normal $(n=26)$ & $98.49 \pm 19.79$ & $88.76 \pm 12.12$ & $0.34 \pm 0.05$ & $0.62 \pm 0.10$ & $5.11 \pm 0.45$ \\
\hline Concentric remodeling $(n=10)$ & $106.44 \pm 3.42$ & $104.94 \pm 2.96$ & $0.51 \pm 0.08$ & $0.76 \pm 0.07$ & $4.52 \pm 0.32$ \\
\hline Concentric hypertrophy $(n=18)$ & $164.81 \pm 24.82$ & $163.35 \pm 34.91$ & $0.56 \pm 0.06$ & $0.74 \pm 0.09$ & $4.96 \pm 0.33$ \\
\hline Eccentric hypertrophy $(n=17)$ & $145.09 \pm 18.17$ & $142.61 \pm 45.98$ & $0.39 \pm 0.04$ & $0.67 \pm 0.09$ & $5.63 \pm 0.61$ \\
\hline
\end{tabular}

N o t e. LVMMI: left ventricle myocardium mass index; RWT: left ventricle relative wall thickness; AVR: atrioventicular ratio; EDD: left ventricle end-diastolic dimension. 
Group 1: patients with normal LV geometry. The group comprised 26 people from 23 to 70 years old (12 men, 14 women). The majority had experienced $\mathrm{AH}$ for less than 10 years $(65.22 \%)$, while fewer had experience it for more than 20 years (13.05\%). The average duration of $\mathrm{AH}$ was 12 years. Average BMl: $28.41 \pm 4.89$. The majority of patients had normal body mass (15.38\%), and the fewest had abdominal obesity (23.08\%). Metabolic syndrome was present in $65.22 \%$ of the patients. These patients had the lowest day-time average SBPs, DBPs and average night-time SBPs, low average night-time DBPs, the lowest pressure-induced load indices by SBP TI within 24 hours, and by DBP TI during the day-time. Cluster analysis demonstrated the greatest percentage of normal BP clusters, and the smallest percentage having ISAH and SDAH.

Group 2: patients with LV concentric remodeling. The group comprised 10 people from 33 to 71 years old (5 men, 5 women). The average AH duration was 18 years. Metabolic syndrome occurred in $77.77 \%$ of the patients in this group. During the day-time, they had the highest indices of pressure-induced load by DBP IT index time, SBP and DBP variability. The average day-time and average night-time BP levels, 24-hour measurements of pressure-induced load by SBP IT, average values of LVMMI, RWT and EDD occupied a position between those groups with normal LV geometry and concentric hypertrophy, but this group was marked by the highest AVR values. Compared to group 1, the occurrence of normal BP in the cluster was lower, while that of SDAH in the cluster was higher.

Group 3: patients with concentric LVH. The group comprised 18 people from 24 to 78 years old $(9$ men, 9 women). As compared to the group with normal LV geometry, the number of patients experiencing $\mathrm{AH}$ for less than 10 years was a factor of 1.2 times smaller $(52.94 \%)$, while the percentage of those experiencing it for more than 20 years was 2.7 times greater $(35.29 \%)$. The average duration of $\mathrm{AH}$ was 17 years. Across the total patient numbers, the highest average BMl was found in this group, the fewest patients had normal body mass $(5.88 \%)$, and the greatest percentage had abdominal obesity $(52.94 \%)$. Metabolic syndrome was present in $87.5 \%$ of these patients.

The development of concentric LVH is associated with high BP and high pressure-induced load. In this group, we found the highest average SBP and DBP values and indices of pressure-induced load by time index: SBP $\mathrm{TI}$ — in both the day-time and night-time; DBP $\mathrm{TI}$ - in the night-time; high DBP TI value - in the day-time; the highest SBP variability - in the day-time, and DBP over the whole $24 \mathrm{~h}$. We revealed the least occurrence of normal BP clusters $(22.14 \%)$ and the greatest occurrence of SDAH clusters ( $48.72 \%)$, including tachycardia during the day-time, apparently due to autonomic imbalance in form of an increase in the activity of the sympathetic nervous system.
The type of hemodynamic overload determines the resulting $\mathrm{LVH}$. In response to chronic pressure-induced overload, and increased afterload, concentric LVH occurs. The increased afterload promotes a rise in LV wall thickness, but without an increase in the size of the cavity, as is evidenced by the highest values of LVMMI, RWT and the lowest average EDDs. Eventually, the elasticity of the LV walls is reduced while their rigidity is increased, LV relaxation ability is disturbed and the restrictive component of intracardiac hemodynamics appears. This is confirmed by the highest AVR indices occurring in the patients with concentric LV remodeling. The results of our investigation confirm the relationship between concentric LVH and high BP. According to conventional 24-hour BPM analysis, the patients with concentric LVH had the highest average values of SBP and DBP, and the highest indices of pressure-induced load and SBP and DBP variability both the during daytime and night-time. According to the cluster analysis, they recorded the highest occurrence of SDAH clusters and the least occurrence of normal BP clusters.

Group 4: patients with eccentric LVH. The group comprised 17 patients from 33 to 69 years old (10 men, 7 women). The group also had the most patients with an $\mathrm{AH}$ duration between 10 and 20 years (23.08\%), while the number of patients with $\mathrm{AH}$ durations of more than 20 years was 2.4 times greater $(30.77 \%)$ than that of the group with normal LV geometry. The average duration of $\mathrm{AH}$ was 15 years. Average BMl exceeded the corresponding measurement in the group of patients with normal LV geometry, but was somewhat less than in those patients with concentric LV remodeling. Metabolic syndrome occurred in $84.61 \%$ of the patients. Certain peculiarities of 24-hour BPM and echoCG comparative analysis are noteworthy.

1. When compared to the group with normal LV geometry:

during the day-time, the average measurements of SBP and DBP are comparable, however, during the night-time they exceed those of the normal LV geometry group;

the pressure-induced load measurements by SBP $\mathrm{TI}$ over $24 \mathrm{~h}$ exceed the corresponding normal LV group measurements, but for the DBP TI, they do not significantly exceed them;

the occurrence of normal BP clusters is lower, while the number of ISAH and IDAH (specifically IDAH) clusters is higher than over the full 24-hours;

the LVMMI, RWT, AVR measurements are higher.

2. When compared to the group with concentric LVH:

the average SBP, DBP values, the measurements of pressure-induced load by SBP TI and the DBP TI, SBP and DBP variability during both day-time and night-time is lower;

the occurrence of normal BP clusters and the number of ISAH and IDAH (specifically IDAH) clusters is higher than over the full 24-hours; 
the LVMMI, RWT, AVR measurements are lower, but the EDD has a higher value.

Concentric remodeling is more frequent in moderate than in soft $\mathrm{AH}$, while eccentric LVH is more frequent in soft $\mathrm{AH}$ [7]. Eccentric LVH, which is manifested in LV dilation without essential wall thickening, develops under increased preload. Due to volume overload of the heart, LV diastolic filling and wall tension increases, sarcomeric cardiac myocyte rows elongate, and the LV cavity expands. As a result, adaptive eccentric LVH is formed and, according to the Frank-Starling law, this provides for the long-term maintenance of cardiac output.

It has now been conclusively proven that myocardial remodeling in $\mathrm{AH}$ is caused not only by elevated $\mathrm{BP}$ but also by damaging overload. According to Makolkin [8], the simultaneous presence of carbohydrate and lipid metabolism disorder, obesity and autonomic imbalance contribute to the development of LVH. Among the humoral disturbances having a net trophic effect on the myocardium, and participating in the remodeling processes, the lead roles belong to pathological activation of the rennin-angiotensin-aldosterone system, the sympathoadrenal system and to hyperinsulinemia. The remodeling process is associated with changes in water-salt metabolism, and with primary or neurohumoral-mediated abnormalities of ion transport within cells.

Our findings suggest that, in patients with eccentric $\mathrm{LVH}$, non-hemodynamic factors are of significant pathogenetic importance for the development of LV remodeling. In this group, as compared to the group of patients with concentric LVH, lower measurements were recorded for average day-time and average nighttime BP, pressure-induced load and BP variability. The average day-time values of SBP, DBP and measurements of pressure-induced load by DBP TI are similar to the measurements in those patients with normal LV geometry, while the average night-time values of SBP, DBP and measurements of pressure-induced load by SBP TI are higher. The group of patients with eccentric LVH recorded the highest occurrence of ISAH and IDAH clusters.

The emergence of ISAH is associated with remodeling of the walls of the great vessels; this aspect of $\mathrm{AH}$ is considered to be, not only a risk factor, but a target lesion marker as well [9]. Increased occurrence of ISAH clusters during the day-time in patients with concentric $\mathrm{LVH}$, and over the full 24-hours in patients with eccentric
$\mathrm{LVH}$, can be deemed to be an unfavorable sign that apparently reflects general processes of cardiovascular system remodeling.

Conclusion. Such an integrated approach to the interpretation of 24-hour blood pressure monitoring results, which comprises both conventional and cluster analysis, allows for objectification of the study results and can reveal significant $\mathrm{AH}$ features in patients with different types of LV remodeling.

Study Funding and Conflicts of Interest. The study was not financed from any sources, and there are no conflicts associated with this study.

\section{References}

1. Russian Medical Society for Arterial Hypertension (RMSAH), Society of Cardiology of Russian Federation (SCRF). Diagnosis and treatment of arterial hypertension. Kardiovaskulyarnaya terapiya i profilaktika 2008; 7(6 Suppl 2).

2. Ostroumova O.D., Shorikova E.G., Galeeva N.Yu. Arterial hypertension and myocardial hypertrophy of left ventricle. Losartan: "old friends are better than new ones". Russkij medicinskij zurnal 2011; 19(4): 200-204.

3. Ratova L.G., Dmitriev V.V., Tolpygina S.N., Chazova I.E. 24-hour blood pressure monitoring in clinical practice. Consilium Medicum 2001; 13: 327-345.

4. Rogoza A.N., Agal'tsov M.V., Sergeeva M.V. Sutochnoe monitorirovanie arterial'nogo davleniya: varianty vrachebnykh zaklyucheniy $\mathrm{i}$ kommentarii [24-hour blood pressure monitoring: medical notes options and comments]. Nizhny Novgorod: DEKOM; 2005.

5. Shkarin V.V. Sistemnyy podkhod $v$ diagnostike, lechenii $i$ vedenii patsientov $s$ arterial'noy gipertenziey $v$ ambulatornykh usloviyakh. Dis. ...dokt. med. nauk [Systematic approach in diagnosis, treatment and arterial hypertension disease management for outpatients. DSc Dissertation]. Nizhny Novgorod; 1999.

6. Ganau A., Devereux R.B., Roman M.J., de Simone G., Pickering T.G., Saba P.S., Vargiu P., Simongini I., Laragh J.H. Patterns of left ventricular hypertrophy and geometric remodeling in essential hypertension. J Am Coll Cardiol 1992; 19(7): 15501558, http://dx.doi.org/10.1016/0735-1097(92)90617-V.

7. Shlyakhto E.V., Konradi A.O., Zakharov D.V., Rudomanov O.G. Structural-functional changes of myocardium in hypertensive disease. Kardiologia 1999; 39(2): 49-55.

8. Makolkin V.I. Metabolicheskiy sindrom IMetabolic syndrome]. Moscow: Meditsinskoe informatsionnoe agentstyo; 2010; $142 \mathrm{p}$.

9. Mukhin A.V., Fomin V.V., Moiseev S.V., et al. Microalbuminuria - an integral marker of cardiorenal relations in arterial hypertension. Consilium Medicum 2007: 9(5), 13-19. 